\title{
Effects of nuclear structure on parity nonconservation in atomic cesium in relativistic mean field theory
}

\author{
P. K. Panda* and B. P. Das \\ Indian Institute of Astrophysics, Koramangala, Bangalore 560 034, India
}

(Received 11 October 1999; published 27 October 2000)

\begin{abstract}
We use relativistic mean field theory, which includes scalar and vector mesons, to calculate the binding energy and charge radii in ${ }^{125} \mathrm{Cs}-{ }^{139} \mathrm{Cs}$. We then evaluate the nuclear structure corrections to the weak charges for a series of cesium isotopes using different parameters and estimate their uncertainty in the framework of this model.

PACS number(s): 24.80.+y, 21.60.-n
\end{abstract}

Experiments suggested in [1] for measuring parity nonconservation (PNC) in heavy atoms have provided an important confirmation $[2,3]$ of the $S U(2) \times U(1)$ electroweak sector of the standard model. One can explore the possibility of new physics beyond the standard model [4] by combining the very accurate measurement of PNC in atomic cesium with sophisticated many-body calculations [5,6]. A recent measurement of parity nonconservation in atomic cesium [3] has reduced significantly the uncertainty $(<1 \%)$ in the determination of the weak charge $Q_{W}$ of the Cs nucleus [3]. The latest result [7] $Q_{W}^{\text {expt }}=-72.06(28)_{\text {expt }}(34)_{\text {atomic theory }}$ disagrees slightly, at the $2.5 \sigma$ level, with the standard model prediction of $Q_{W}^{s t}{ }^{\text {model }}=-73.12 \pm 0.06$. The experimental result needs input from atomic structure calculations in the vicinity of the nucleus. However, the small but nonnegligible effects of nuclear size must be addressed before an interpretation of PNC data in terms of the fundamental electroweak couplings is possible. In particular, variations in the neutron distribution among the isotopes affect the weak charge. Thus nuclear structure could become a crucial factor in the interpretation of PNC experiments of increasing accuracy $[8-11]$.

There have been earlier studies to determine nuclear structure effects in PNC in atomic cesium using nonrelativistic potentials $[10,11]$. Recently a parametric approach of nuclear effects in atomic parity nonconservation has been used [12]. In this paper we present a relativistic calculation of these effects for the first time using relativistic mean field (RMF) theory.

The RMF theory first proposed by Teller and co-workers $[13,14]$ and Dürr [15] and later by Walecka [16] and developed by others has been fairly successfully applied to both finite nuclear matter and infinite nuclei. RMF theory has the advantage that, with proper relativistic kinemetics and with the mesons and their properties already known or fixed from the properties of a small number of nuclei [17-21], the method gives a good description for binding energies, root mean square (rms) radii, quadrupole and hexadecapole deformations, and other nuclear properties not only spherical,

\footnotetext{
*Present address: Instituto de Física Teorica, Universidade Estadual Paulista, Rua Pamplona 145, 01405-900 Sao Paulo - SP, Brazil. Electronic address: panda@ift.unesp.br
}

but also for deformed nuclei. The same parameter set of the model also describes well the properties of nuclear matter. One of the major attractive features of the RMF approach is that the spin-orbit interaction and the associated nuclear shell structure automatically arise from the meson-nucleon interaction. The inclusion of the $\rho$ meson accounts for the neutron-proton asymmetry. We can therefore expect the RMF calculation to provide useful information on nuclear structure corrections to atomic parity nonconservation.

In the standard model, the electron-nucleon interaction is mediated by both the photon and the intermediate boson $Z^{0}$. The latter does not conserve parity. The energy involved in atomic PNC experiments is usually only a fraction of an eV, while the mass of the $Z^{0}$ is $\simeq 92 \mathrm{GeV}$, and so the parity nonconserving interaction may be written as a contact interaction. We have

$$
\begin{aligned}
H_{p n c}= & \frac{G_{F}}{\sqrt{2}} \sum_{e B}\left[C_{1 B} \int \psi_{B}^{\dagger} \psi_{B} \psi_{e}^{\dagger} \gamma_{5} \psi_{e} d^{3} r\right. \\
& \left.+C_{2 B} \int \psi_{B}^{\dagger} \sigma_{B} \psi_{B} \cdot \psi_{e}^{\dagger} \mathbf{a} \psi_{e} d^{3} r\right],
\end{aligned}
$$

where $B$ stands for $n$ (neutron) or $p$ (proton). The first term grows coherently with nucleon numbers $N$ and $Z$. The second term together with the anapole moment term amounts to at most a few percent of the first term in heavy atoms. We shall therefore consider only the first term. The effective Hamiltonian becomes

$$
\begin{aligned}
H_{p n c}= & \frac{G_{F}}{2 \sqrt{2}} \int\left[-N \rho_{n}(\mathbf{r})+Z(1\right. \\
& \left.\left.-4 \sin ^{2} \theta_{W}\right) \rho_{p}(\mathbf{r})\right] \psi_{e}^{\dagger} \gamma_{5} \psi_{e} d^{3} r,
\end{aligned}
$$

where the proton and neutron densities $\rho_{p, n}(\mathbf{r})$ are normalized to unity. We have assumed the standard model nucleon couplings

$$
\begin{gathered}
C_{1 p} \equiv 2 C_{1 u}+C_{1 d}=\frac{1}{2}\left(1-4 \sin ^{2} \theta_{W}\right), \\
C_{1 n} \equiv 2 C_{1 u}+C_{1 d}=-\frac{1}{2} .
\end{gathered}
$$


TABLE I. Parameters.

\begin{tabular}{lccc}
\hline \hline & TM1 & NL1 & NL-SH \\
\hline$M$ & 938.0 & 938.0 & 939.0 \\
$m_{\sigma}$ & 511.198 & 492.25 & 526.059 \\
$m_{\omega}$ & 783.0 & 795.359 & 783.0 \\
$m_{\rho}$ & 770.0 & 763.0 & 763.0 \\
$g_{\sigma}$ & 10.0289 & 10.138 & 10.444 \\
$g_{2}$ & -7.2325 & -12.172 & -6.9099 \\
$g_{3}$ & 0.6183 & -36.265 & -15.8337 \\
$g_{\omega}$ & 12.6139 & 13.285 & 12.945 \\
$c_{3}$ & 71.3075 & 0.000 & 0.000 \\
$g_{\rho}$ & 4.6322 & 4.9755 & 4.383 \\
\hline \hline
\end{tabular}

We need the spatial variation of the electron part $\psi_{e}^{\dagger} \gamma_{5} \psi_{e}$ over the nucleus, its normalization, and its dependence on nuclear structure. PNC effects are dominated by $s$ electrons $(\kappa=-1)$ coupled to $p$ electrons $(\kappa=1)$. This can be expressed as

$$
\rho_{5}(r) \equiv \psi_{p}^{\dagger} \gamma_{5} \psi_{s}=C(Z) \mathcal{N}(Z, R) f(r)
$$

where $C(Z)$ contains all atomic structure effects for a point nucleus including many-body correlations, $\mathcal{N}$ $\equiv \psi_{p}^{\dagger}(0) \gamma_{5} \psi_{s}(0)$ is the normalization factor for a single electron, and $f(r)$ describes the spatial variation [normalized such that $f(0)=1]$. It is the integrals

$$
q_{n, p}=\int f(r) \rho_{n, p}(r) d^{3} r
$$

which determine the effect of the proton and neutron distributions on the PNC observables.

Observable PNC effects are proportional to the matrix element between two atomic states $i$ and $j$,

$$
\mathcal{O}=\left\langle i\left|H_{p n c}\right| j\right\rangle=\frac{G_{F}}{2 \sqrt{2}} C Z \mathcal{N}\left[Q_{W}(N, Z)+Q_{W}^{n u c}(N, Z)\right],
$$

where $Q_{W}(N, Z)$ is the weak charge. For the standard model, the weak charge takes the form

$$
Q_{W}(N, Z)=-N+Z\left(1-4 \sin ^{2} \theta_{W}\right) .
$$

The nuclear structure correction $Q_{W}^{n u c}(N, Z)$ describes the part of the PNC effect that arises from the finite nuclear size. In the same approximation as Eq. (8) above,

$$
Q_{W}^{n u c}(N, Z)=-N\left(q_{n}-1\right)+Z\left(1-4 \sin ^{2} \theta_{W}\right)\left(q_{p}-1\right) .
$$

The proton (charge) nuclear form factors needed for $q_{p}$ and $\mathcal{N}$ are generally well known from measurements of the charge distribution of nuclei close to the stable valley and many unstable nuclei as well. The neutron nuclear form factor needed for $q_{n}$ is not well determined experimentally and is model dependent. To estimate the importance of PNC in nuclear structure, the form factor can be approximated to the order of $(Z \alpha)^{2}$ for a sharp nuclear surface and neglecting the electron mass in comparison with the nuclear Coulomb potential [9],

$$
f(r) \simeq 1-\frac{1}{2}(Z \alpha)^{2}\left[(r / R)^{2}-\frac{1}{5}(r / R)^{4}+\frac{1}{75}(r / R)^{6}\right] .
$$

In the above, for a sharp nuclear surface density distribution, the only relevant parameter is the nuclear radius $R$ and $\left\langle r^{2 n}\right\rangle=3 /(2 n+3) R^{2 n}$.

The relativistic Lagrangian density for a nucleon-meson many-body system [17-20] is

$$
\begin{aligned}
\mathcal{L}= & \bar{\psi}_{i}\left(i \gamma^{\mu} \partial_{\mu}-M\right) \psi_{i} \\
& +\frac{1}{2} \partial^{\mu} \sigma \partial_{\mu} \sigma-\frac{1}{2} m_{\sigma}^{2} \sigma^{2}+\frac{1}{3} g_{2} \sigma^{3}+\frac{1}{4} g_{3} \sigma^{4}-g_{s} \bar{\psi}_{i} \psi_{i} \sigma \\
& -\frac{1}{4} \Omega^{\mu \nu} \Omega_{\mu \nu}+\frac{1}{2} m_{\omega}^{2} \omega^{\mu} \omega_{\mu}+\frac{1}{4} c_{3}\left(\omega_{\mu} \omega^{\mu}\right)^{2} \\
& -g_{\omega} \bar{\psi}_{i} \gamma^{\mu} \psi_{i} \omega_{\mu}-\frac{1}{4} \vec{B}^{\mu \nu} \cdot \vec{B}_{\mu \nu}+\frac{1}{2} m_{\rho}^{2} \vec{R}^{\mu} \cdot \vec{R}_{\mu} \\
& -g_{\rho} \bar{\psi}_{i} \gamma^{\mu} \vec{\tau} \psi_{i} \cdot \vec{R}^{\mu}-\frac{1}{4} F^{\mu \nu} F_{\mu \nu}-e \bar{\psi}_{i} \gamma^{\mu} \frac{\left(1-\tau_{3 i}\right)}{2} \psi_{i} A_{\mu} .
\end{aligned}
$$

TABLE II. Results of RMF calculations in the TM1 parameter set. The binding energies are in MeV; all radii are in $\mathrm{fm}$.

\begin{tabular}{lccccccccc}
\hline \hline$N$ & $E$ & $E_{R M F}$ & $r_{c h}$ & $r_{c h}(\mathrm{fitted}){ }^{\mathrm{a}}$ & $\delta r_{p}^{2}(\mathrm{expt})$ & $\delta r_{p}^{2}$ & $\delta r_{n}^{2}$ & $\delta r_{p}^{4}$ & $\delta r_{n}^{4}$ \\
\hline 70 & 1049.98 & 1054.856 & 4.800 & 4.784 & -0.1517 & -0.2373 & -1.1301 & 10.8178 & 54.5746 \\
72 & 1068.25 & 1073.06 & 4.807 & 4.789 & -0.0985 & -0.1709 & -0.8281 & 7.7116 & 40.2391 \\
74 & 1085.66 & 1090.754 & 4.813 & 4.794 & -0.0561 & -0.114 & -0.5438 & 5.1508 & 26.5791 \\
76 & 1102.37 & 1107.645 & 4.819 & 4.798 & -0.0141 & -0.057 & -0.2749 & 2.5803 & 13.1588 \\
78 & 1118.52 & 1121.879 & 4.825 & 4.800 & 0.0000 & 0.0000 & 0.0000 & 0.0000 & 0.0000 \\
80 & 1134.24 & 1139.389 & 4.831 & 4.801 & 0.0250 & 0.0571 & 0.2391 & 2.59 & 11.8781 \\
82 & 1149.27 & 1154.266 & 4.843 & 4.808 & 0.0821 & 0.1716 & 0.58 & 7.7995 & 29.6266 \\
84 & 1159.57 & 1164.659 & 4.863 & 4.837 & 0.3604 & 0.3727 & 1.0448 & 17.0111 & 52.7265 \\
\hline \hline
\end{tabular}

${ }^{\mathrm{a}}$ References [23]. 
TABLE III. Results of RMF calculations in the NL1 parameter set. The binding energies are in MeV; all radii are in fm.

\begin{tabular}{lccccccccc}
\hline \hline$N$ & $E$ & $E_{R M F}$ & $r_{c h}$ & $r_{c h}(\mathrm{fitted})^{\mathrm{a}}$ & $\delta r_{p}^{2}(\mathrm{expt})$ & $\delta r_{p}^{2}$ & $\delta r_{n}^{2}$ & $\delta r_{p}^{4}$ & $\delta r_{n}^{4}$ \\
\hline 70 & 1049.98 & 1053.328 & 4.813 & 4.784 & -0.1517 & 0.038 & -1.1299 & 6.013 & 55.5158 \\
72 & 1068.25 & 1071.530 & 4.811 & 4.789 & -0.0985 & -0.1521 & -0.9041 & 6.8677 & 44.6223 \\
74 & 1085.66 & 1088.718 & 4.816 & 4.794 & -0.0561 & -0.0951 & -0.5979 & 4.3004 & 29.6961 \\
76 & 1102.37 & 1105.499 & 4.822 & 4.798 & -0.0141 & -0.0476 & -0.2899 & 2.1536 & 14.4871 \\
78 & 1118.52 & 1121.167 & 4.827 & 4.800 & 0.0000 & 0.0000 & 0.0000 & 0.0000 & 0.0000 \\
80 & 1134.24 & 1136.719 & 4.831 & 4.801 & 0.0250 & 0.0381 & 0.2412 & 1.7296 & 12.1809 \\
82 & 1149.27 & 1152.056 & 4.839 & 4.808 & 0.0821 & 0.1143 & 0.5545 & 5.1964 & 28.1745 \\
84 & 1159.57 & 1157.077 & 4.855 & 4.837 & 0.3604 & 0.2769 & 1.1457 & 12.6254 & 58.8962 \\
\hline \hline
\end{tabular}

${ }^{\mathrm{a}}$ References [23].

The field for the $\sigma$ meson is denoted by $\sigma$, that of the $\omega$ meson by $\omega_{\mu}$, and that of the isovector $\rho$ meson by $\vec{R}_{\mu}$. Here $A^{\mu}$ denotes the electromagnetic field. $\psi_{i}$ are the Dirac spinors for the nucleons, whose third component of isospin is denoted by $\tau_{3 i}$. Here $g_{s}, g_{\omega}, g_{\rho}$, and $e^{2} / 4 \pi=\frac{1}{137}$ are the coupling constants for $\sigma, \omega$, and $\rho$ mesons and photons, respectively. $M$ is the mass of the nucleon and $m_{\sigma}, m_{\omega}$, and $m_{\rho}$ are the masses of the $\sigma, \omega$, and $\rho$ mesons, respectively. $\Omega^{\mu \nu}, \vec{B}^{\mu \nu}$, and $F^{\mu \nu}$ are the field tensors for the $\omega^{\mu}, \vec{R}^{\mu}$, and the photon fields, respectively [19]. The field equations for mesons and nucleons are obtained from the Lagrangian of Eq. (11) and can be found in Refs. [18,19]. These are nonlinear, coupled partial differential equations, which are solved self-consistently. These equations are solved by expanding the upper and lower components $f^{ \pm}$and $g^{ \pm}$of the Dirac spinors $\psi_{i}$ and the boson field wave functions in terms of a deformed harmonic oscillator potential basis. The frequencies $\hbar \omega_{\perp}$ and $\hbar \omega_{z}$ can be expressed in terms of the deformation parameter $\beta_{0}$. In numerical calculations, we have used the maximum number of oscillator quanta, $N_{\max }$ $=16$, for both fermions and bosons.

The total binding energy of the system is

$$
E_{\text {tot }}=E_{\text {part }}+E_{\sigma}+E_{\omega}+E_{\rho}+E_{C}+E_{\text {pair }}+E_{\text {c.m. }},
$$

where $E_{\text {part }}$ is the sum of single-particle energies of the nucleons, $E_{\sigma}, E_{\omega}$, and $E_{\rho}$ are the contributions of meson energies, and $E_{C}$ and $E_{\text {pair }}$ are the Coulomb and pairing energy, respectively. We have used the pairing gap defined in Ref. [22] to take pairing in to account. $E_{\text {c.m. }}=$ $-\frac{3}{4} 41 A^{-1 / 3}$ is the nonrelativistic approximation for the center-of-mass correction.

The three-vector part of the vector meson fields does not respect time reversal symmetry due to the odd $Z$ number of Cs isotopes. In this RMF calculation, we take into account the nucleon current and the resulting spatial component of the vector field due to $\omega$ mesons, and we have neglected the contributions of the spatial components of the vector fields of $\rho$ mesons and photons.

We use the parameter sets TM1, NL1, and NL-SH for our calculations. These parameters are given in Table I. We note that TM1 parameter set has the non-negative value of the quartic self-coupling coefficient $g_{3}$. In most of the successful parameter sets the quartic self-coupling term is negative, so that the energy spectrum is unbounded below, although in normal cases the solutions are obtained in the local minimum. However, all these parameter sets give a good account of various properties such as binding energy, compressibility, and asymmetric energy for nuclear matter.

For an open shell nuclei, i.e., ${ }^{133} \mathrm{Cs}$, the pairing may be important. Therefore the binding energies $r_{p}$ and $r_{n}$ are calculated where the effect of pairing is incorporated in the constant gap approximation. In Tables II, III, and IV, the binding energies, charge radius, and shifts $\delta r_{p, n}^{2}$ and $\delta r_{p, n}^{4}$ are listed with different parameter sets. The binding energies agree in all the cases with the experimental values with

TABLE IV. Results of RMF calculations in the NL-SH parameter set. The binding energies are in MeV; all radii are in fm.

\begin{tabular}{lccccccccc}
\hline \hline$N$ & $E$ & $E_{R M F}$ & $r_{c h}$ & $r_{c h}(\mathrm{fitted})^{\mathrm{a}}$ & $\delta r_{p}^{2}(\mathrm{expt})$ & $\delta r_{p}^{2}$ & $\delta r_{n}^{2}$ & $\delta r_{p}^{4}$ & $\delta r_{n}^{4}$ \\
\hline 70 & 1049.98 & 1055.723 & 4.773 & 4.784 & -0.1517 & -0.1981 & -1.044 & 8.8134 & 49.6998 \\
72 & 1068.25 & 1072.937 & 4.779 & 4.789 & -0.0985 & -0.1416 & -0.7633 & 6.4073 & 35.1781 \\
74 & 1085.66 & 1091.900 & 4.784 & 4.794 & -0.0561 & -0.0945 & -0.5102 & 4.2116 & 24.5616 \\
76 & 1102.37 & 1107.703 & 4.789 & 4.798 & -0.0141 & -0.0473 & -0.2558 & 2.1091 & 12.3785 \\
78 & 1118.52 & 1124.199 & 4.794 & 4.800 & 0.0000 & 0.0000 & 0.0000 & 0.0000 & 0.0000 \\
80 & 1134.24 & 1141.868 & 4.799 & 4.801 & 0.0250 & 0.0473 & 0.2373 & 2.1157 & 11.6014 \\
82 & 1149.27 & 1158.148 & 4.811 & 4.808 & 0.0821 & 0.161 & 0.5755 & 7.2211 & 28.3272 \\
84 & 1159.57 & 1166.610 & 4.829 & 4.837 & 0.3604 & 0.3417 & 1.077 & 15.3843 & 53.554 \\
\hline \hline
\end{tabular}

${ }^{\mathrm{a}}$ References [23]. 
TABLE V. The weak charges $Q_{W}(N, Z)$, nuclear structure corrections $Q_{W}^{n u c}(N, Z), q_{n}, q_{p}$ (including intrinsic nucleon-structure correction), $R$ (in fm), and $R_{n} / R_{p}$ in the TM1 parameter set.

\begin{tabular}{ccccccc}
\hline \hline$N$ & $Q_{W}(N, Z)$ & $Q_{W}^{n u c}(N, Z)$ & $q_{n}$ & $q_{p}$ & $R$ & $R_{n} / R_{p}$ \\
\hline 70 & -65.2174 & 2.9327 & 0.9552 & 0.958 & 6.1968 & 1.026 \\
72 & -67.1930 & 3.0497 & 0.9548 & 0.958 & 6.2058 & 1.031 \\
74 & -69.1686 & 3.1682 & 0.9544 & 0.958 & 6.2135 & 1.036 \\
76 & -71.1442 & 3.2808 & 0.9541 & 0.958 & 6.2213 & 1.0404 \\
78 & -73.1198 & 3.3946 & 0.9538 & 0.958 & 6.229 & 1.0448 \\
80 & -75.0954 & 3.5096 & 0.9535 & 0.958 & 6.2368 & 1.0485 \\
82 & -77.0710 & 3.6258 & 0.9532 & 0.958 & 6.2525 & 1.0529 \\
84 & -79.0466 & 3.7514 & 0.9528 & 0.958 & 6.2781 & 1.0579 \\
\hline \hline
\end{tabular}

maximum deviation of 5-6 MeV out of a total binding energy of $1000 \mathrm{MeV}$. The charge radii $R_{c h}$ agree with the fitted values [23] to within $1 \%$. The agreement of the shift $\delta r_{p}^{2}$ with experiment is not very good. Such a failure to reproduce the radii is also observed in $\mathrm{Sk} M^{*}$ and $\mathrm{SkmIII}$ interaction calculations. It is worthwhile to make a brief comment about the comparison with the values of $\left\langle r_{p}^{2}\right\rangle$. Experimentally, muonic $\mathrm{X}$-ray energies for stable ${ }^{133} \mathrm{Cs}$ have been fitted by the Fermi distribution with the halfway radius $\mathrm{c}=5.85 \mathrm{fm}$, surface thickness $t=1.82$ fm $[24,25]$, and $\left\langle r_{p}^{2}\right\rangle$ $=23.27 \mathrm{fm}^{2}$. The RMF calculation gives $\left\langle r_{p}^{2}\right\rangle=22.63$ in TM1, $\left\langle r_{p}^{2}\right\rangle=22.65$ in NL1, and $\left\langle r_{p}^{2}\right\rangle=22.34$ in NL-SH parameters.

The calculated shifts in neutron radii are listed in Table II (TM1), III (NL1), and IV (NL-SH). The lack of unambiguous precise experimental information on the neutron distribution means that one must extrapolate to the desired neutron properties. In Ref. [26], data from pionic atoms are analyzed. The nearest nucleus to cesium in Ref. [26] is ${ }^{142} \mathrm{Ce}$. Scaling it with $A^{2 / 3}$, one obtains $\left\langle r_{n}^{2}\right\rangle=24.7 \mathrm{fm}^{2}$ for ${ }^{133} \mathrm{Cs}$. The RMF calculation gives $24.71 \mathrm{fm}^{2}$ (TM1), 25.13 $\mathrm{fm}^{2}$ (NL1), and $24.32 \mathrm{fm}^{2}$ (NL-SH), respectively. We note that there is essentially no model-independent experimental information on neutron density distributions. Thus, our calculation cannot be verified directly. We next use these radii to estimate the nuclear structure effects in PNC.

One of the motivations for further improving atomic parity nonconservation experiments is to test the standard model parameters. To show how they might compare in size to nuclear structure effects, we begin by rewriting Eqs. (8) and (9) in the form

$$
\begin{aligned}
& Q_{W}(N, Z)= 0.9878 \times[-N+Z(1-4.0118 \bar{x})] \\
& \times(1.0+0.00782 T), \\
& \bar{x}=0.23124 \pm 0.00017+0.003636 S-0.00258 T,
\end{aligned}
$$

where $\bar{x}$ is assumed here to be defined at the mass scale $M_{Z}$ by modified minimal subtraction [27,28], $S$ is the parameter characterizing the isospin conserving new quantum loop cor-
TABLE VI. Same as Table V in the NL1 parameter set.

\begin{tabular}{ccccccc}
\hline \hline$N$ & $Q_{W}(N, Z)$ & $Q_{W}^{n u c}(N, Z)$ & $q_{n}$ & $q_{p}$ & $R$ & $R_{n} / R_{p}$ \\
\hline 70 & -65.2174 & 2.9466 & 0.9550 & 0.958 & 6.2135 & 1.0283 \\
72 & -67.1930 & 3.0853 & 0.9543 & 0.958 & 6.211 & 1.0375 \\
74 & -69.1686 & 3.2048 & 0.9539 & 0.958 & 6.2174 & 1.0427 \\
76 & -71.1442 & 3.3184 & 0.9536 & 0.958 & 6.2252 & 1.0482 \\
78 & -73.1198 & 3.4409 & 0.9532 & 0.958 & 6.2316 & 1.0531 \\
80 & -75.0954 & 3.5570 & 0.9529 & 0.958 & 6.2368 & 1.0573 \\
82 & -77.0710 & 3.6825 & 0.9525 & 0.958 & 6.2471 & 1.062 \\
84 & -79.0466 & 3.8261 & 0.9519 & 0.958 & 6.2678 & 1.0703 \\
\hline \hline
\end{tabular}

rections, and $T$ is the parameter characterizing isospin breaking corrections. The nuclear structure correction to $Q_{W}$ is given by

$$
\begin{aligned}
Q_{W}^{n u c}(N, Z)= & 0.9878 \times\left[-N\left(q_{n}-1\right)\right. \\
& \left.+Z(1-4.0118 \bar{x})\left(q_{p}-1\right)\right] .
\end{aligned}
$$

The coefficients $q_{n, p}$ defined earlier in Eq. (6) contain the nuclear structure effects. We have included the intrinsic nucleon structure contributions in evaluating the nuclear structure correction. We use [10]

$$
q_{p, n}=\int d^{3} r\left[\rho_{p, n}(r)+\frac{1}{6}\left\langle r^{2}\right\rangle_{I,(p, n)} \Delta^{2} \rho_{p, n} / Q_{p, n}^{W}\right] f(r),
$$

where $\left\langle r^{2}\right\rangle_{I,(p, n)}$ are the nucleon weak radii and $Q_{p, n}^{W}$ are nucleon weak charges. Including the strangeness radii [29] of the nucleon $\left(0.14 \mathrm{fm}^{2}\right)$, the nuclear structure corrections and the weak charge for different isotopes of cesium evaluated for $S=T=0$ are listed in Tables V (TM1), VI (NL1), and VII (NL-SH) for different parameter sets. Here one can see that the $q_{p}$ are constant when the neutron number increases from $N=70$ to 84 . However, $q_{n}$ varies slowly as one increases the neutron number. Our RMF calculation gives the nuclear structure correction for ${ }^{133} \mathrm{Cs}, Q_{W}^{n u c}=3.3946$ for TM1, 3.4409 for NL1, and 3.3869 for NL-SH forces. Similar corrections were also obtained in the nonrelativistic potentials (3.422 for $\mathrm{SkM}^{*}$ and 3.458 for SkmIII) [11]. In Fig. 1,

TABLE VII. Same as Table V in the NL-SH parameter set.

\begin{tabular}{ccccccc}
\hline \hline$N$ & $Q_{W}(N, Z)$ & $Q_{W}^{n u c}(N, Z)$ & $q_{n}$ & $q_{p}$ & $R$ & $R_{n} / R_{p}$ \\
\hline 70 & -65.2174 & 2.9327 & 0.9552 & 0.958 & 6.1619 & 1.0253 \\
72 & -67.1930 & 3.0497 & 0.9548 & 0.958 & 6.1697 & 1.0301 \\
74 & -69.1686 & 3.1609 & 0.9545 & 0.958 & 6.1761 & 1.0345 \\
76 & -71.1442 & 3.2733 & 0.9542 & 0.958 & 6.1826 & 1.0389 \\
78 & -73.1198 & 3.3869 & 0.9539 & 0.958 & 6.1890 & 1.0433 \\
80 & -75.0954 & 3.5014 & 0.9536 & 0.958 & 6.1954 & 1.0473 \\
82 & -77.0710 & 3.6258 & 0.9532 & 0.958 & 6.2109 & 1.0518 \\
84 & -79.0466 & 3.7514 & 0.9528 & 0.958 & 6.2342 & 1.0581 \\
\hline \hline
\end{tabular}




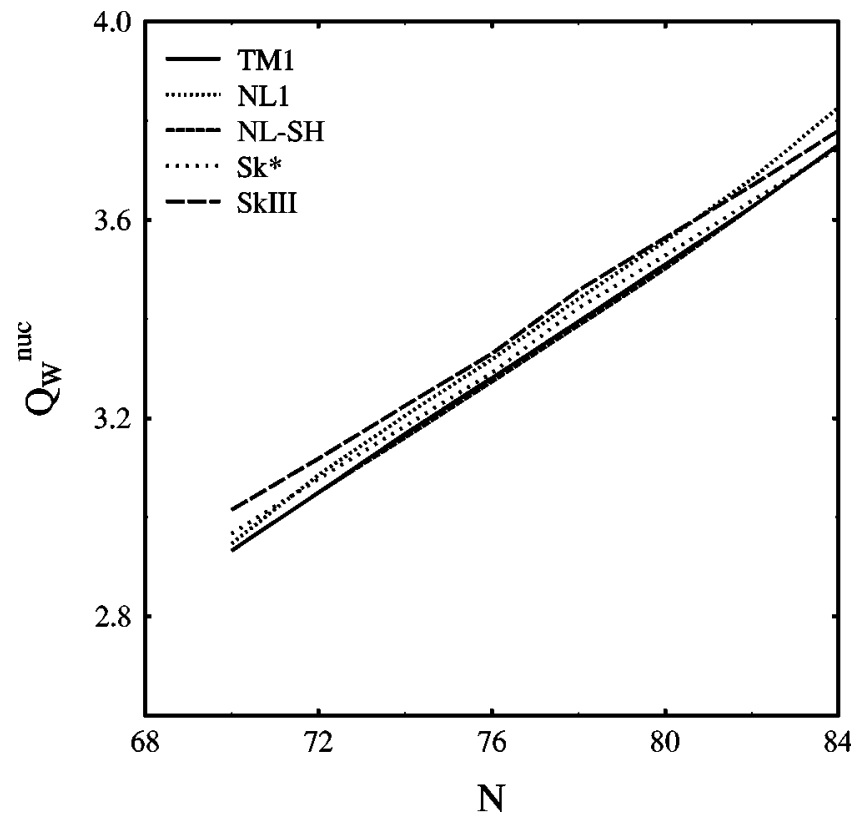

FIG. 1. $N$ vs $Q_{W}^{n u c}$ for cesium isotopes in different parameter sets.

we have plotted the nuclear structure corrections versus different isotopes of cesium for the parameter sets used in our calculations.

We next discuss explicitly the correction to the weak charge arising from the difference between the neutron and proton distributions. The small difference between $q_{n}$ and $q_{p}$ has the effect of modifying the effective weak charge as [30]

$$
Q_{W}=Q_{W}^{s t .}{ }^{m o d}+\Delta Q_{W}^{n-p},
$$

where

$$
\Delta Q_{W}^{n-p}=N\left(1-q_{n} / q_{p}\right)
$$

Assuming the difference by a small parameter, $R_{n}^{2} / R_{p}^{2}=1$ $+\epsilon$, we have

$$
\Delta Q_{W}^{n-p} \simeq N(Z \alpha)^{2}(0.221 \epsilon) / q_{p} .
$$

Our RMF calculation gives $\Delta Q_{W}^{n-p}=0.2656$ in TM1, $\Delta Q_{W}^{n-p}=0.3161$ in NL1, and $\Delta Q_{W}^{n-p}=0.2565$ in NL-SH parameters. The Skyrme parametrized nuclear Lagrangian (SkM* ${ }^{*}$ and SkIII) yields $R_{n} / R_{p}$ values of 1.022 and 1.016, respectively, which gives $\Delta Q_{W}^{n-p}=0.11$. The RMF calculations generate larger neutron radii and thus predict values of $\Delta Q_{W}^{n-p}$ that are at least twice as large as those obtained from the Skyrme interaction. However, if one wants to attribute the small differences between the experimental value of the $Q_{W}$ and the standard model prediction entirely to nuclear physics effects, one would require $R_{n} / R_{p}=1.18 \pm 0.07$ (adding all atomic experimental and theoretical and standard model theoretical errors), which is beyond the range of all predicted nuclear structure values.

The uncertainty in $\Delta Q_{W}^{n-p}$ for our calculations is now closer to the atomic theory uncertainty than previous calculations. It is therefore necessary to improve the uncertainties in both nuclear and atomic structure if one would like to use $\mathrm{PNC}$ in atomic cesium as a probe of physics beyond the standard model.

Note added. After completion of this work, there appeared an article by Vretenar, Lalazissis, and Ring [31], in which the authors present $R_{n} / R_{p}=1.046$ for ${ }^{133}$ Cs in the NL3 parameter set in relativistic mean field theory. They have also estimated that the $\Delta Q_{W}^{n-p}$ can be more than a factor of 2 larger than that calculated in [30].

The authors acknowledge financial support of the Department of Atomic Energy (No 37/15/97/-R\&D. II/1603). The services and computer (Power-Challenge RS10000) facilities provided to us by the Indian Institute of Astrophysics are gratefully acknowledged. The authors are thankful to S.J. Pollock and M.C. Welliver for useful discussions.
[1] M.A. Bouchiat and C. Bouchiat, Phys. Lett. 48B, 111 (1974); I.B. Khriplovich, Pis'ma Zh. Éksp. Teor. Fiz 20, 686 (1974) [JETP Lett. 20, 315 (1974)]; P.G.H. Sandars, in Atomic Physics, edited by G. zu Pulitz (Plenum, New York, 1975), Vol. 4, p. 71; D.S. Sorede and E.N. Fortson, Bull. Am. Phys. Soc. 20, 491 (1975).

[2] N.H. Edwards, S.J. Phipp, P.E.G. Baird, and S. Nakayama, Phys. Rev. Lett. 74, 2654 (1995); D.M. Meekhof, P.K. Majumder, S.K. Lamoreaux, and E.N. Forston, Phys. Rev. A 52, 1895 (1995); M.J.D. Macpherson, K.P. Zetie, R.B. Warrington, D.N. Stacey, and J.P. Hoare, Phys. Rev. Lett. 67, 2784 (1991).

[3] C.S. Wood, S.C. Bennett, D. Cho, B.P. Masterson, J.L. Roberts, C.E. Tanner, and C.E. Wieman, Science 275, 1759 (1997).

[4] P. Langacker, M.-X. Luo, and A.K. Mann, Rev. Mod. Phys. 64, 87 (1992).

[5] V.A. Dzuba, V.V. Flambaum, and O.P. Sushkov, Phys. Lett. A 141, 147 (1989).

[6] S.A. Blundell, W.R. Johnson, and J. Sapirstein, Phys. Rev.
Lett. 65, 1411 (1990).

[7] S.C. Bennett and C.E. Wieman, Phys. Rev. Lett. 82, 2484 (1999).

[8] C.E. Wieman, C. Monroe, and E.A. Cornel, in Laser Spectroscopy X, edited by H. Henrikson and P. Vogel (World Scientific, Singapore, 1990).

[9] E.N. Fortson, Y. Pang, and L. Wilets, Phys. Rev. Lett. 65, 2857 (1990).

[10] S.J. Pollock, E.N. Fortson, and L. Wilets, Phys. Rev. C 46, 2587 (1992).

[11] B.Q. Chen and P. Vogel, Phys. Rev. C 48, 1392 (1993).

[12] J. James and P.G.H. Sandars, J. Phys. B 32, 3295 (1999).

[13] H.P. Dürr and E. Teller, Phys. Rev. 101, 494 (1956).

[14] M.H. Johnson and E. Teller, Phys. Rev. 98, 783 (1955).

[15] H.P. Dürr, Phys. Rev. 103, 469 (1956).

[16] J.D. Walecka, Ann. Phys. (N.Y.) 83, 491 (1974).

[17] B.D. Serot and J.D. Walecka, Adv. Nucl. Phys. 16, 1 (1986).

[18] P.G. Reinhard, M. Rufa, J. Maruhn, W. Greiner, and J. Friedrich, Z. Phys. A 323, 13 (1986). 
[19] M. Rufa, P.-G. Reinhard, J.A. Maruhn, W. Greiner, and M.R. Strayer, Phys. Rev. C 38, 390 (1988); P.-G. Reinhard, Z. Phys. A 329, 257 (1988); Y.K. Gambhir, P. Ring, and A. Thimet, Ann. Phys. (N.Y.) 198, 132 (1990); M.M. Sharma, M.A. Nagarajan, and P. Ring, Phys. Lett. B 312, 377 (1993).

[20] J. Boguta and A.R. Bodmer, Nucl. Phys. A292, 413 (1977).

[21] Y. Sugahara and H. Toki, Nucl. Phys. A579, 557 (1994).

[22] P. Moller et al., At. Data Nucl. Data Tables 39, 225 (1988).

[23] E.G. Nadjakov, K.P. Marinova, and Ya.P. Gangrsky, At. Data Nucl. Data Tables 56, 133 (1994).

[24] R. Engfer et al., At. Data Nucl. Data Tables 14, 509 (1974).
[25] W.Y. Lee et al., Phys. Rev. Lett. 23, 648 (1969).

[26] C. Garcia-Recio, J. Nieves, and E. Oset, Nucl. Phys. A547, 473 (1992).

[27] J. Erler and P. Langacker, Eur. J. Phys. 1, 90 (1998).

[28] W.J. Marciano and J.L. Rosner, Phys. Rev. Lett. 65, 2963 (1990).

[29] R.L. Jaffe, Phys. Lett. B 229, 275 (1989).

[30] S.J. Pollock and M.C. Welliver, Phys. Lett. B 464, 177 (1999).

[31] D. Vretenar, G.A. Lalazissis, and P. Ring, Phys. Rev. C 62, 045502 (2000). 